POWEREDBYSCIENCE@DIRECT®

Pattern Recognition Letters 25 (2004) 73-86
Pattern Recognition Letters

www.elsevier.com/locate/patrec

\title{
An invariant scheme for exact match retrieval of symbolic images based upon principal component analysis
}

\author{
D.S. Guru *, P. Punitha \\ Department of Studies in Computer Science, University of Mysore, Manasagangothri, Mysore 570006, Karnataka, India
}

Received 12 June 2003; received in revised form 21 August 2003

\begin{abstract}
In this paper, an important aspect in creating a symbolic image database (SID), useful for exact match retrieval is addressed. The problem in conventional pairwise spatial relationships (particularly the 9DLT matrix) based approach for representing symbolic images in SID is discussed. An efficient method specifically for exact match retrieval, invariant to image transformations is proposed. In order to take care of image transformations, a new concept called direction of reference is introduced. The relative spatial relationships existing among the components present in an image are perceived with respect to the direction of reference and preserved by a set of triples. The proposed method is based upon principal component analysis (PCA). The first principal component vector (PCV) of the set of triples corresponding to an image is computed and stored as the representative of that image. The PCVs corresponding to $n$ images to be archived in the SID are stored in a sorted order. A methodology for exact match retrieval is also presented in this paper. The presented retrieval algorithm takes $\mathrm{O}(\log n)$ search time in the worst case, with the help of the binary search technique, where $n$ is the number of symbolic images stored in the SID.
\end{abstract}

(c) 2003 Elsevier B.V. All rights reserved.

Keywords: Symbolic image; Direction of reference; Relative spatial relationship; Principal component vector; Symbolic image database; Binary search; Exact match retrieval

\section{Introduction}

Retrieval of images with the desired content from a symbolic image database (SID) is a challenging and motivating research issue. A SID is a system in which a large amount of picture data and their related information are represented by both

\footnotetext{
${ }^{*}$ Corresponding author. Tel.: +91-821-2415355; fax: +91821-2510789.

E-mail addresses: guruds@mailcity.com,guruds@lycos.com (D.S. Guru), punithaswamy@yahoo.com (P. Punitha).
}

logical images and physical images. A logical image, also referred as symbolic image, can be regarded as an abstract physical image, while the physical image is the real image itself. However, to effectively represent/retrieve an image in/from a SID, the attributes such as symbols/icons and their relationships which are rich enough to describe the corresponding symbolic image are necessary. Thus, many researchers (Chang et al., 1987, 1989; Chang and $\mathrm{Wu}, 1992 ; \mathrm{Wu}$ and Chang, 1994; Huang and Jean, 1994; Zhou and Ang, 1997; Zhou et al., 2001) have highlighted the importance of perceiving 
spatial relationships existing among the components of an image for efficient representation/ retrieval of symbolic images in/from a SID. Infact, the perception of spatial relationships preserves the reality being embedded in physical images besides making the system intelligent, fast and flexible.

Basically, there are two kinds of retrieval: similarity retrieval and exact match retrieval. In similarity retrieval, the task is to retrieve from the SID, all those images that are similar to a given query image, while the exact match retrieval process retrieves from the SID only those images exactly identical (i.e., 100\% similar) to a query image.

Infact, exact match retrieval is a special case of similarity retrieval and more precisely is an image recognition problem. Exact match retrieval is widely used in professional applications on industrial automation, biomedicine, social security, crime prevention, medical diagnosis and prevention and many more robotics/multi-spectral/computer vision applications.

There have been several attempts made by the research community to scatter the demands in the design of efficient, invariant, flexible and intelligent image archival and retrieval systems based on perception of spatial relationships. To make image retrieval, visualization, and traditional image database operations more flexible and faster, data structure should be object oriented. The design of such object oriented algorithms began with the discovery of 2D string (Chang et al., 1987) representation. Based on the 2D string representation, many algorithms were proposed (Chang and $\mathrm{Li}$, 1988; Jungert, 1988; Chang et al., 1989; Lee and Hsu, 1990, 1991; Chang and Lin, 1996; Chang and Ann, 1999) to represent symbolic images in a SID.

Using the concept of 2D string, in order to retrieve similar symbolic images from a SID, algorithms (Lee et al., 1989; Lee and Shan, 1990; Lee and Hsu, 1992) based on the longest common subsequence matching were also proposed. Though, these iconic image representation schemes offer many advantages, the linear string representation given to the spatial relations existing among the components takes non-deterministic-polynomial time complexity during the process of string matching and is not invariant to image transformations, especially to rotation. In order to reduce the search time and to avoid string matching, hash oriented methodologies for similarity retrieval based upon the variations of $2 \mathrm{D}$ string were explored (Chang and $\mathrm{Wu}, 1992 ; \mathrm{Wu}$ and Chang, 1994; Bhatia and Sabharwal, 1994; Sabharwal and Bhatia, 1995, 1997). However, hash function based algorithms require $\mathrm{O}\left(\mathrm{m}^{2}\right)$ search time in the worst case for retrieval of symbolic images, where $m$ is the number of iconic objects.

Chang (1991) proposed a symbolic indexing approach called nine directional lower triangular (9DLT) matrix to encode symbolic images. A 9DLT matrix was used to preserve the spatial relationships existing among objects in a symbolic image. As a result of this, three models for image archival and retrieval were developed (Chang and Jiang, 1994; Chang and Wu, 1995; Zhou and Ang, 1997).

Chang and $\mathrm{Wu}$ (1995) proposed a scheme based on 9DLT matrix for exact match retrieval. Indeed, to the best of our knowledge, that is the only model proposed for exact match retrieval. In their work, the pairwise spatial relationships existing between iconic objects were preserved with the help of nine directional codes and were then represented in a 9DLT matrix. The first principal component direction of the set of triples representing the 9DLT matrix of a symbolic image was computed and stored as the representative of the symbolic image in the SID. The first principal component direction of all symbolic images were stored in a sorted sequence, there by enabling the retrieval process to consume only $\mathrm{O}(\log n)$ search time even in the worst case, with the help of the binary search technique, where $n$ is the number of symbolic images stored in the SID. This is the most efficient and the only method proposed so far for the retrieval of symbolic images with $\mathrm{O}(\log n)$ search time complexity. Despite its incomparable efficiency, the method is not robust to take care of image transformations especially, rotation.

However, one can find the existence of a few invariant models (Petraglia et al., 1996; Zhou et al., 2001) in literature for similarity retrieval. Although, these invariant models proposed for similarity retrieval can be used for exact match retrieval, it is not advisable due to the fact that the exact match retrieval can be achieved more efficiently and more effectively with less comput- 
ational effort and less resource investment when compared to that of similarity match retrieval. Hence, design of an efficient, effective and invariant model for exact match retrieval still remains as an open issue in the field of image databases.

In view of this, an efficient method specifically for exact match retrieval, invariant to image transformations is proposed in this paper. In order to take care of image transformations, a new concept called direction of reference is introduced. The direction of reference is conceptually aligned with the $X$-axis of the co-ordinate system and the spatial relationships existing among the objects present in the image are perceived relative to the direction of reference and then are preserved by the use of triples. The proposed method is based upon principal component analysis (PCA). The first principal component vector (PCV) of the set of triples corresponding to an image is computed and stored as the representative of that image. The PCVs corresponding to $n$ images to be archived in the SID are stored in a sorted order. The proposed retrieval algorithm requires $\mathrm{O}(\log n)$ search time in the worst case during retrieval, with the help of the binary search technique, where $n$ is the number of symbolic images stored in the SID. Though the proposed model appears to be an improvement over Chang and Wu's (1995) model, it is altogether a new model. The proposed method preserves the actual orientation angle which is computed relative to the direction of reference, while Chang and Wu's (1995) model is based on the nine directional codes.

The remaining part of the paper is organized as follows. Section 2 gives an overview of the exact match retrieval approach. Section 3 discusses the major problem with the 9DLT matrix based approaches. In Section 4, an invariant methodology for exact match retrieval based on the novel concept of direction of reference is proposed. The results of the experiments conducted to establish the efficacy of the proposed methodology are given in Section 5. Section 6 follows with discussion and conclusion.

\section{An overview of exact match retrieval scheme}

Before giving details on the exact match retrieval (Chang and $\mathrm{Wu}, 1995)$ procedure, initially in this section, a simple example to illustrate the concept of 9DLT matrix is given. This is followed by a brief description of the exact match retrieval scheme (Chang and $\mathrm{Wu}, 1995)$.

\subsection{Nine directional lower triangular (9DLT) matrix}

Consider a symbolic image consisting of four components with labels $L_{1}, L_{2}, L_{3}$ and $L_{4}$ as shown in Fig. 1. We may use nine directional codes shown in Fig. 2 to represent the pairwise spatial relationships between $x$, a referenced component and $y$, a contrasted component. The directional code say $r=0$, represents that $y$ is to the east of $x, r=1$ represents that $y$ is to the north-east of $x$, and so on. Thus, the 9DLT matrix $T$ for the symbolic image of Fig. 1 is as shown in Fig. 3. Since each

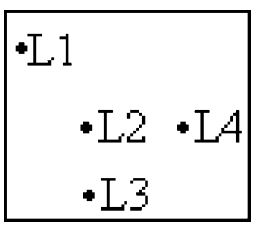

Fig. 1. A symbolic image.

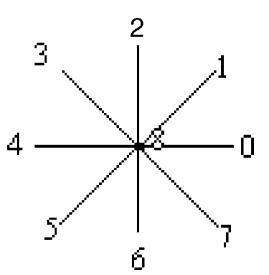

Fig. 2. The nine directional codes.

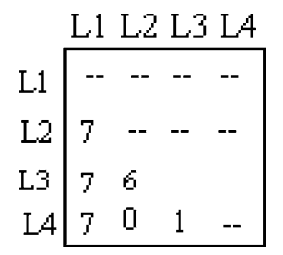

Fig. 3. The 9DLT matrix of Fig. 1. 
relationship is represented by a single triple $(x, y, r)$ the 9DLT matrix is a lower triangular matrix.

The 9DLT matrix can now be formally defined as follows (Chang, 1991). Let $V=\left\{v_{1}, v_{2}, v_{3}\right.$, $\left.v_{4}, \ldots, v_{m}\right\}$ be a set of $m$ distinct components/ objects. Let $Z$ consist of ordered components $z_{1}, z_{2}, z_{3}, \ldots, z_{s}$ such that, $\forall i=1,2, \ldots, s, z_{i} \in V$. Let $C$ be the set of nine directional codes as defined in Fig. 2. Each directional code is used to specify the spatial relationship between two components. So, a 9DLT matrix $T$ is an $s \times s$ matrix over $C$ in which $t_{i j}$, the $i$ th row and $j$ th column element of $T$ is the directional code of $Z_{j}$ to $Z_{i}$ if $j<i$, and undefined otherwise. The matrix $T$ is a 9DLT matrix according to the ordered set $Z$.

\subsection{Exact match retrieval scheme (Chang and $W u$, 1995)}

Using the concept of 9DLT matrix, Chang and $\mathrm{Wu}$ (1995) proposed an exact match retrieval scheme based upon PCA. The 9DLT matrix (Fig. 3 ) is represented by a set of triples, $\left\{\left(L_{1}, L_{2}, 7\right)\right.$, $\left.\left(L_{1}, L_{3}, 7\right),\left(L_{1}, L_{4}, 7\right),\left(L_{2}, L_{3}, 6\right),\left(L_{2}, L_{4}, 0\right),\left(L_{3}, L_{4}, 1\right)\right\}$ or simply $\{(1,2,7),(1,3,7),(1,4,7),(2,3,6),(2,4$, $0),(3,4,1)\}$. The first PCV, $(-0.1977,-0.1568$, 0.9676), of the above set of triples was found and stored in SID as the representative of the symbolic image.

Thus, the retrieval of a symbolic image requires one to:

- Construct the 9DLT matrix for the given symbolic image;

- Find out the first PCV (say $D$ ) of the set of triples representing the 9DLT matrix (algorithm to find out the first PCV of the set of samples, in general, is given in Appendix A);

- Search for $D$ in SID;

- Extract the image index associated with $D$.

\section{Problems in 9DLT matrix based approach}

Let us assume that a rotated version (Fig. 4) of the symbolic image shown in Fig. 1 is given as input during the retrieval phase. For the sake of simplicity, the angle of rotation is taken, in this

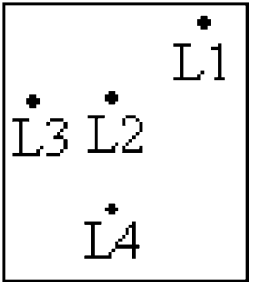

Fig. 4. A rotated version of Fig. 1.

example, as $-90^{\circ}$. The corresponding 9DLT matrix of the rotated symbolic image is shown in Fig. 5 and the corresponding set of triples is $\{(1,2,5)$, $(1,3,5),(1,4,5),(2,3,5),(2,4,6),(3,4,7)\}$. The associated first PCV is $(0.5048,0.4705,0.7237)$. It is clearly observed that the first PCVs associated with 9DLT matrices shown in Figs. 3 and 5 are not identical, as their corresponding sets of triples are totally different even though they represent the same physical image. This problem is due to the fact that the directional codes are not invariant to rotation. Instead of considering the pairwise spatial relationships directly between two components independent of other components present in the image, if the relationship is perceived relative to another iconic object, then the problem of getting different interpretations for the symbolic images representing the same physical image in different orientations perhaps could be resolved. An invariant model to perceive relative spatial relationship between two iconic objects is thus proposed and explained in the next section.

\section{The proposed methodology}

The proposed scheme has two stages. The first stage proposes a novel method for representing

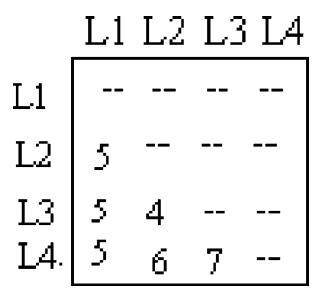

Fig. 5. The 9DLT matrix of Fig. 4. 
images invariant to image transformations in the SID, while the second stage suggests a corresponding exact match retrieval scheme for a given query image invariant to image transformations.

\subsection{Representation of images in SID}

The proposed representation scheme computes for each image, a direction of reference with respect to which the pairwise spatial relationships existing among the components of the image are recommended to be perceived and preserved by the use of a set of triples. Subsequently, the PCA is employed to give a compact representation to the set of triples obtained. Thus, the following are the major steps involved in the proposed representation scheme.

\subsubsection{Computation of direction of reference}

It is understood that, instead of perceiving the pairwise spatial relationship directly between two components, if it is perceived relative to a direction called direction of reference, which is unique to an image, then the problem of getting different interpretations for the symbolic images of the same physical image in different orientations could be resolved. Perhaps, one such direction could be the direction of the line segment joining the centre of an image to the centroid of the iconic object which is nearer to the centre of the image. However, consideration of such a line segment as the line of reference is not advisable since it is not always possible to acquire images with the same scene coverage. Thus, we recommend to choose a directed line segment connecting two distinct iconic objects present in an image, which are at the farthest distance as the line of reference, and its direction from the object with lesser iconic label to the other object as the direction of reference of the image.

Formally, let $I$ be an image consisting $m$ number of iconic objects. Let $O_{1}, O_{2}, O_{3}, \ldots, O_{k}$ be $k \leqslant m$ distinct iconic objects present in $I$ and let $L_{1}, L_{2}, L_{3}, \ldots, L_{k}$ be their respective labels such that, $L_{i}<L_{j} ; i, j=1,2, \ldots, k$ and $i<j$. Let $O_{p}$ and $O_{q}$ be two iconic objects with $L_{p}<L_{q}$, which are at the farthest distance.

$$
\text { i.e., } \begin{aligned}
\operatorname{dist}\left(O_{p}, O_{q}\right) & =\max \left\{\operatorname{dist}\left(O_{i}, O_{j}\right) \forall i, j\right. \\
& \left.\in\{1,2, \ldots, k\} \text { and } L_{i} \neq L_{j}\right\}
\end{aligned}
$$

where, dist( ) is a distance function which computes the Euclidean distance between two iconic objects. The line joining the iconic object $O_{p}$ to $O_{q}$ is the line of reference and its direction from the object $O_{p}$ to the object $O_{q}$ is the direction of reference for the image $I$.

If $\left(x_{p}, y_{p}\right)$ and $\left(x_{q}, y_{q}\right)$ are the co-ordinates of the centroids of $O_{p}$ and $O_{q}$ respectively, then we define,

$\alpha=\tan ^{-1}\left(\frac{y_{q}-y_{p}}{x_{q}-x_{p}}\right)$

and

$\beta=\sin ^{-1}\left(\frac{y_{q}-y_{p}}{\operatorname{dist}\left(O_{p}, O_{q}\right)}\right)$

The direction of the line joining $O_{p}$ to $O_{q}$ is given by,

$\theta= \begin{cases}\alpha+\pi & \text { if } \alpha<0 \text { and } \beta>0 \\ \alpha-\pi & \text { if } \alpha>0 \text { and } \beta<0 \\ \alpha & \text { otherwise }\end{cases}$

Fig. 6 shows an image consisting of a total of $m=6$ instances of $k=4$ distinct iconic objects with the direction of reference from $O_{p}$ to $O_{q}$ where $p=2$ and $q=3$.

An image sometimes may have more than one pair of distinct objects placed at the same farthest distance resulting with more than one candidate for line of reference. If such is the situation, then the conflict in choosing a unique direction of reference for such images is resolved by choosing the

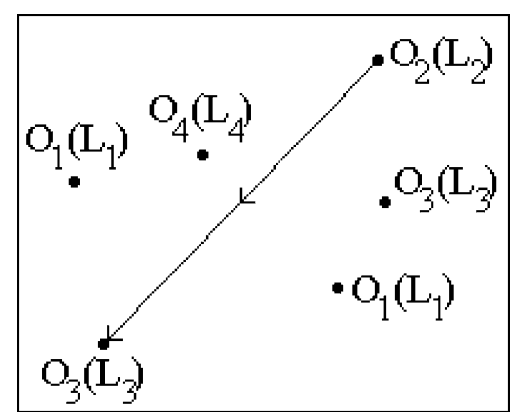

Fig. 6. An image with direction of reference $(m=6, k=4)$. 


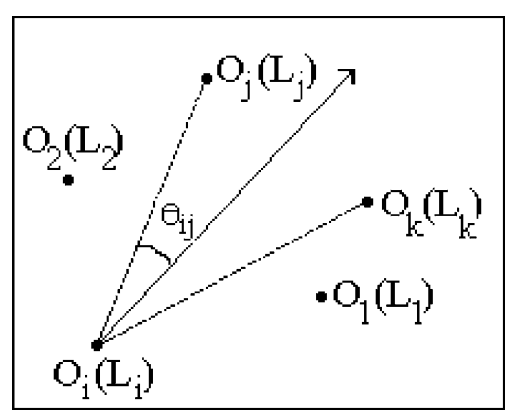

Fig. 7. Perception of spatial relationship relative to the direction of reference.

line segment obtained by triangular law of addition of all the candidates for line of reference as the line of reference, and its direction as the direction of reference of the image. Fig. 7 shows an instance for the above situation. The object pairs $\left(O_{i}, O_{j}\right)$ and $\left(O_{i}, O_{k}\right)$ are at the same farthest distance. Therefore, the two line segments (shown in dotted lines in Fig. 7) connecting the object $O_{i}$ to $O_{j}$ and $O_{i}$ to $O_{k}$ are the candidates for line of reference. The direction of reference of the image is the direction of the line segment (line with an arrow) obtained by adding the candidates for line of reference using triangular law of addition of vectors (here each line segment is a vector).

\subsubsection{Perception of spatial relationship}

Once the direction of reference $\theta$ is computed, the relative pairwise spatial relationships between each pair of objects are perceived with respect to the direction of reference. In order to make the system invariant to image transformations, the direction of reference is conceptually aligned with that of the positive $X$-axis of the coordinate system. This rotation invariant property could even be achieved by rotating the image itself by the angle $-\theta$, but it is not advisable as rotating an image consumes much time and results with distortion.

If $O_{i}$ and $O_{j}$ are two iconic objects such that, $L_{i}<L_{j}$, as shown in Fig. 7, then the spatial relationship $\theta_{i j}$ of the contrast object $O_{j}$ to the reference object $O_{i}$, is the relative direction of the line segment joining $O_{i}$ and $O_{j}$, with respect to the di- rection of reference $\theta$ and it is preserved by the use of a triple $\left(L_{i}, L_{j}, \theta_{i j}\right)$. On the other hand, if $L_{i}=L_{j}$, then the spatial relationship $\theta_{i j}$ and $\theta_{j i}$ are computed and the direction of the line segment joining $O_{i}$ and $O_{j}$, is the one which forms a lesser angle with respect to the direction of reference. Thus, there are $T=m(m-1) / 2$ number of triples generated to logically represent the image consisting $m$ number of iconic objects.

\subsubsection{Creation of symbolic image database}

Let $S$ be the set of triples generated for a symbolic image as explained in the previous subsection. The set $S$ therefore logically represents the symbolic image and can itself be stored in the SID for the matching process at the time of retrieval. However, it could be unwieldy as the size of $S$ in general is $\mathrm{O}\left(\mathrm{m}^{2}\right)$, where $m$ is the total number of components in the symbolic image. In order to minimize the storage requirement, we suggest to find the first PCV, $D$, the vector on which the variance of the corresponding projected points is maximally preserved after projecting the triples of $S$ onto $D$, assuming that the set $S$ is a set of threedimensional samples, each of which is represented as a point in three-dimensional space. Hence, PCA is employed on $S$ and its first PCV, $D$ is found. This vector $D$ can be stored in the SID to represent the symbolic image. Thus, the creation of a SID involves computation of the first PCV for each image to be archived and then storing them in a sorted sequence so that the binary search technique could be employed. This helps in minimizing the search time in addition to reduction in the size of the SID.

In spite the method is theoretically claimed to be invariant, due to the limitations of the computing system in handling floating point numbers and also because of rotation errors, the components of the PCV cannot be expected to remain entirely invariant but to lie within a certain range. Since the PCV has three values, each rotated instance of an image can be looked upon as a point in three-dimensional Euclidean space $R^{3}$. Therefore, the set of all rotated instances of an image defines in this manner a subspace of $R^{3}$ and the centroid of that subspace is chosen as the representative of the image in SID. 
The following algorithm has thus, been devised to create a SID for a given set of symbolic images useful for exact match retrieval.

Algorithm: Creation_of_SID
Input: Set of Symbolic images
Output: SID, Symbolic Image Database
Method:
$\quad$ For each symbolic image $I$ do
$\quad$ For each rotated instance $R_{I}$ of $I$ do

(a) Compute the direction of reference $\theta$.

(b) Perceive the relative pairwise spatial relationship between each pair of iconic objects in $R_{I}$ with respect to the direction of reference $\theta$ and work out the corresponding set of triples $S$.

(c) Compute the first principal component vector $D$ of $S$ (see Appendix A).

For end

(d) Compute the representative vector $C_{I}$ (for the image $I$ ) which is the centroid

For end of all $D$ s computed for $I$.

(e) Store the representative vectors obtained for all images in a sorted sequence.

\section{Creation_of_SID ends.}

Based on the above representation scheme, a methodology for exact match retrieval is devised and is as simple as described in the next section.

\subsection{Proposed exact match retrieval scheme}

Exact match retrieval is an image retrieval process where a symbolic image $I$ is retrieved as an exact match to a given query image $Q$, if and only if both $I$ and $Q$ are identical. Since each symbolic image $I$ is represented in terms of a vector, which is the average of the PCVs computed for all rotated instances of $I$, the retrieval process reduces to a problem of searching for, if not an exact, a nearest neighbor for the computed vector $D$ of the query image $Q$, in the SID. Since the vectors in SID are stored in a sorted order, the desired image can be retrieved in $\mathrm{O}(\log n)$ search time, by employing the modified binary search algorithm (Guru et al., 2000) where, $n$ is the number of images stored in the SID. The modified binary search technique searches for two successive vectors which bound $D$ in SID. Once such successive vectors are found, their distances to $D$ are computed and the image corresponding to the vector which is nearer to $D$ is retrieved from the SID as the desired image.

Thus, we propose the following algorithm to retrieve images from the SID based on exact match.

Algorithm: Symbolic_image_retrieval

Input: $Q$, Query symbolic image

SID, Symbolic Image Database

Output: Desired image

Method:

(a) Compute the direction of reference for $Q$

(b) Compute the set of triples $S_{q}$ preserving the pairwise spatial relationships among the components present in $Q$, relative to the direction of reference.

(c) Find out the first principal component vector say, $D_{q}$ of $S_{q}$.

(d) Employ the modified binary search technique to find out two adjacent vectors $D_{i}$ and $D_{i+1}$ such that $D_{i} \leqslant D_{q} \leqslant D_{i+1}$.

(e) Find out the distances, say $d_{1}$ and $d_{2}$ of $D_{q}$ to $D_{i}$ and $D_{i+1}$ respectively.

(f) Retrieve the image corresponding to the index $\begin{cases}i & \text { if } d_{1}<d_{2} \\ i+1 & \text { otherwise }\end{cases}$

Symbolic_image_retrieval ends.

\section{Experimental results}

To corroborate the efficacy of the proposed methodology, we have conducted several experiments on various symbolic images of both model images and real images. Out of them we present only three experiments here.

\subsection{Experimentation 1}

We have conducted an experiment on the standard symbolic images (see Fig. 8(f1)-(f4)) considered by Chang and $\mathrm{Wu}$ (1995). During representation, each symbolic image is considered in 76 different orientations, out of which 72 are rotated instances (at $5^{\circ}$ regular interval) and 4 are 


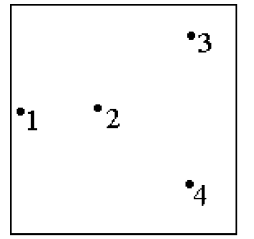

f 1

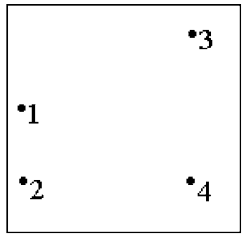

f 2

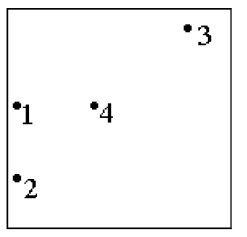

f 3

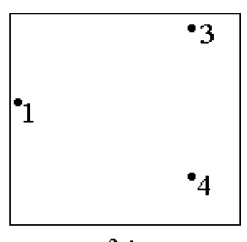

f 4

Fig. 8. Symbolic images taken from Chang and Wu (1995).

scaled versions with $\pm 10 \%$ and $\pm 20 \%$ scaling factors. For each instance of a symbolic image, a direction of reference is computed as explained in Section 4.1.1. The pairwise spatial relationships existing between each pair of components are computed with respect to the direction of reference and are represented by a set of triples.

For instance, the direction of reference obtained for the symbolic image shown in Fig. 8(f1) is $0^{\circ}$. The set of triples preserving the pairwise spatial relationship among the components of the symbolic image (Fig. 8(f1)) with respect to the direction of reference is $S_{0}=\{(1,2,0),(1,3,20)$, $(1,4,339),(2,3,35),(2,4,325),(3,4,270)\}$. As there are four components, the set $S_{0}$ has got $C_{2}^{4}=6$ triples. The set $S_{0}$ represents logically the symbolic image (Fig. 8(f1)). The set $S_{0}$ itself could have been stored in SID, but storing the set $S_{0}$ itself is unwieldy as the size of $S_{0}$, in general, is $\mathrm{O}\left(\mathrm{m}^{2}\right)$, where $m$ is the total number of components present in an image. In order to minimize the storage requirement, we compute the first PCV, $D_{0}=$ $(0.00182150,0.00458399,0.99998784)$ of the set of triples $S_{0}$. Likewise, the sets $S_{1}, S_{2}, S_{3}, \ldots, S_{75}$ corresponding to the remaining 75 instances of the symbolic image (Fig. 8(f1)) are generated and the corresponding first PCVs $D_{1}, D_{2}, D_{3}, \ldots, D_{75}$ are computed. Table 1 gives the span in PCV components computed in this manner for all four symbolic images shown in Fig. 8(f1)-(f4). As suggested in Section 4.1.3, we computed the centroids and stored them as the representatives of the respective images in SID (see Table 2) in a sorted sequence. One can notice that the centroids are distinct.

Let the symbolic image shown in Fig. $9(Q)$ be the query image. This query image is an arbitrarily rotated instance, which is not considered during

Table 1

Span in first PCVs for the symbolic images shown in Fig. 8

\begin{tabular}{llcl}
\hline Image index & Span in & \\
\cline { 2 - 4 } & First component & Second component & Third component \\
\hline f1 & 0.00180978 to 0.00183291 & 0.00456946 to 0.00459049 & 0.99998778 to 0.99998790 \\
f2 & -0.00212963 to -0.00209936 & 0.00183430 to 0.00187654 & 0.99999601 to 0.99999607 \\
f3 & -0.00317698 to -0.00315228 & -0.00080662 to -0.00077621 & 0.99999464 to 0.99999470 \\
f4 & 0.00212023 to 0.00216356 & 0.00335688 to 0.00337728 & 0.99999195 to 0.99999213 \\
\hline
\end{tabular}

Table 2

Representative vectors of the symbolic images shown in Fig. 8 in a sorted sequence

\begin{tabular}{llcl}
\hline Image index & First component & Second component & Third component \\
\hline f3 & -0.00316463 & -0.00079141 & 0.99999467 \\
f2 & -0.0021145 & 0.00185542 & 0.99999604 \\
f1 & 0.00182135 & 0.00457998 & 0.99998784 \\
f4 & 0.00214189 & 0.00336708 & 0.99999204 \\
\hline
\end{tabular}




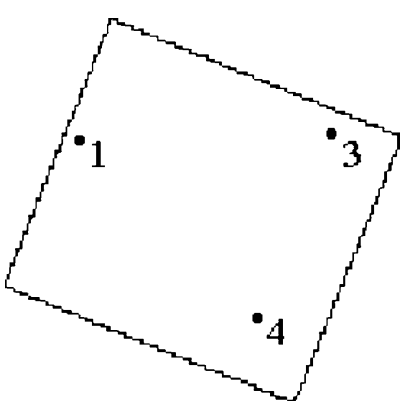

Fig. 9. $Q-$ A rotated instance of Fig. 8(f4).

representation, of the symbolic image shown in Fig. 8(f4). The direction of reference is computed as explained earlier in Section 4.1.1 and the set of triples preserving the spatial relationships existing among the components are generated relative to the direction of reference. The first PCV $D_{q}=$ $(0.00214440,0.00337728,0.99999201)$ is then computed for the set of triples. The modified binary search technique is employed to search, for two successive vectors which bound $D_{q}$. It is found that the vector $D_{q}$ lies in between the representative vectors of the images $\mathrm{f} 1$ and $\mathrm{f} 4$ and their distances from $D_{q}$ are respectively 0.00124534 and 0.00001050 . Since the distance to $\mathrm{f} 4$ is lesser than that of $\mathrm{fl}$, the image $\mathrm{f} 4$ is retrieved and hence $\mathrm{f} 4$ is the desired image.

\subsection{Experimentation 2}

We have considered the symbolic images (Fig. 10) of four keys extracted from (Guru, 2000) and computed the representative vectors shown in Table 3. Similar to that of the previous experimentation, we have considered 76 different instances for each image during representation. We have intentionally chosen this set of images to establish that the method is highly reliable in retrieving the desired image even though the images (Fig. 10) almost look alike or are made up of same number of objects with almost same spatial scattering. Infact, sometimes, even our vision system fails in distinguishing some of the rotated instances of these symbolic images.

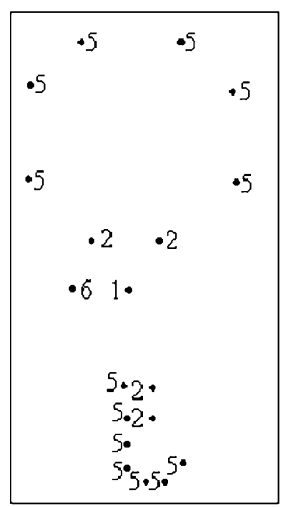

(a)

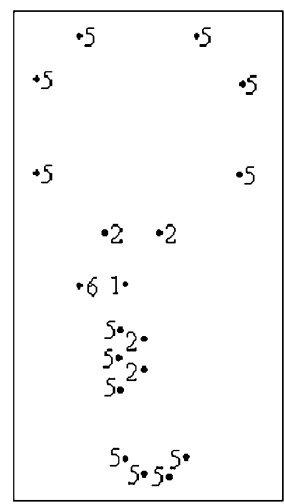

(b)

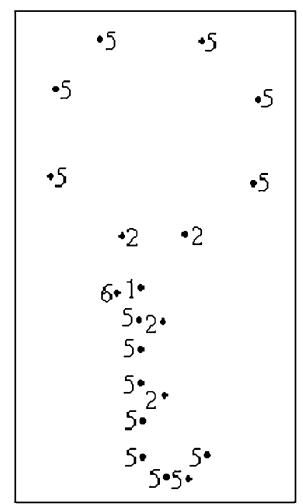

(c)

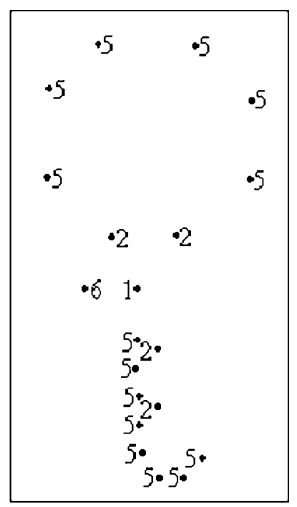

(d)

Fig. 10. Symbolic images of four real keys taken from Guru (2000).

Table 3

Representative vectors for the symbolic images shown in Fig. 10 in a sorted sequence

\begin{tabular}{llll}
\hline Image index & First component & Second component & Third component \\
\hline $\mathrm{c}$ & -0.00827159 & -0.00031386 & 0.99996576 \\
$\mathrm{~d}$ & -0.00810757 & -0.00033761 & 0.99996707 \\
$\mathrm{a}$ & -0.00752611 & -0.00031277 & 0.99997163 \\
$\mathrm{~b}$ & -0.00143076 & -0.00094487 & 0.99999854 \\
\hline
\end{tabular}


The robustness of the proposed retrieval scheme is validated by conducting several experiments on rotated instances of the symbolic images (Fig. 10(a)-(d)) and it is observed that the desired images are retrieved for all query images.

\subsection{Experimentation 3}

Symbolic images in Fig. 11 are extracted from (Guru, 2000). They are the symbolic images obtained for 10 planar objects. In this experiment also, 76 different instances are generated for each image during representation. The representative vectors computed for the images (Fig. 11(P0) (P9)) are as shown in Table 4. One should note that all vectors in Table 4 are distinct except (P6) and (P9) as (P6) is a rotated instance of (P9). The SID is created by eliminating such duplicates and storing them in a sorted sequence. Thus, the modified binary search technique could be employed to search for the vector in SID which is nearer to the first PCV of the query image.
In this experimentation also, we have tested the efficacy of our retrieval scheme with several rotated instances of the symbolic images (Fig. 11) and obtained the desired results.

\subsection{Experimentation 4}

The efficacy of the proposed methodology in retrieving an appropriate image has been further examined by storing the representative vectors of all the images (union of all images considered for experimentation 1,2 and 3) in a single database. All the representative vectors are stored in a sorted sequence and is given in Table 5. This database is created by considering a total of 1368 instances of the symbolic images (Figs. 8, 10 and 11). It can be noticed that the representative vectors corresponding to the images are distinct and unique. It is also observed that the performance of the proposed retrieval scheme is accurate in retrieving the appropriate image of interest, for a given query image.

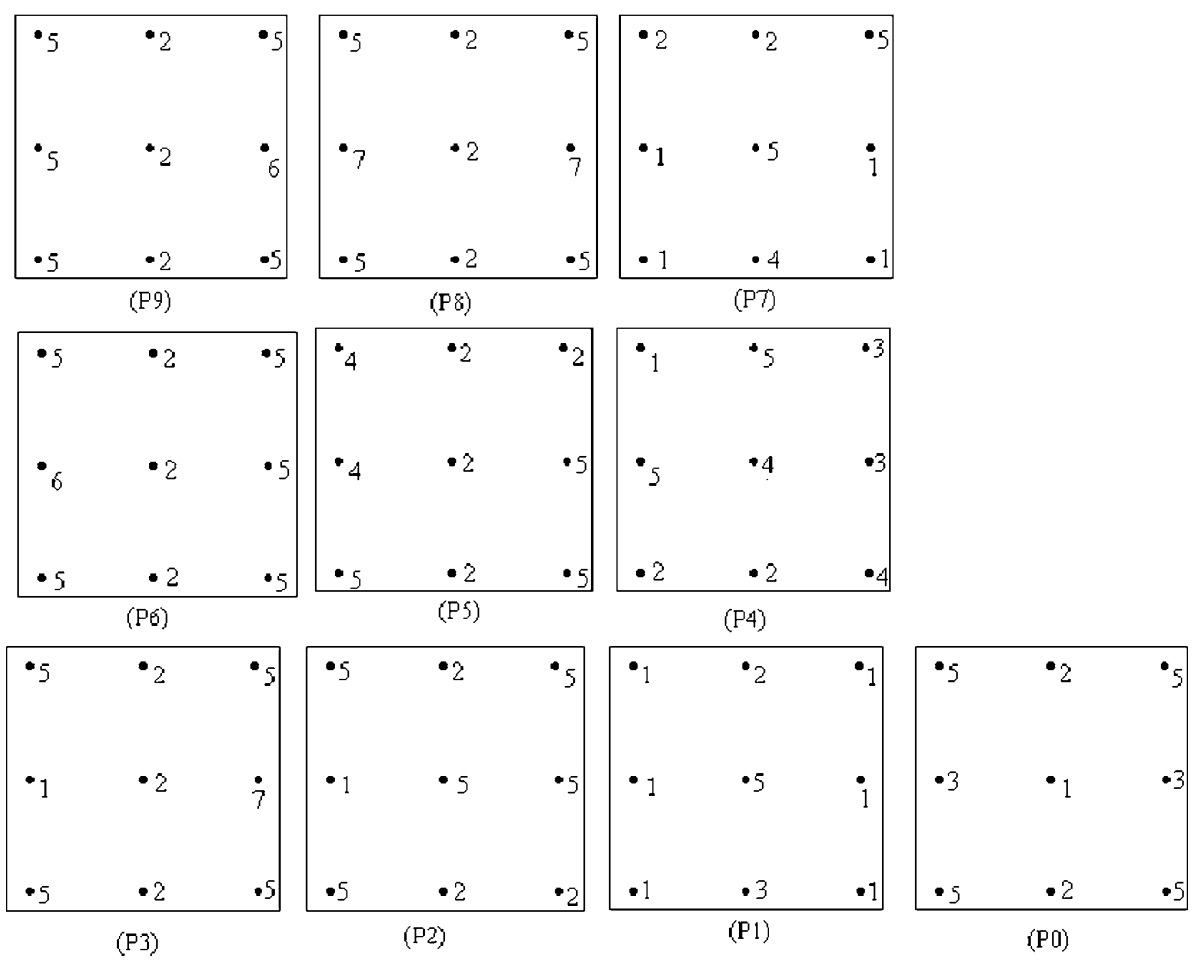

Fig. 11. Symbolic images of 10 planar objects taken from Guru (2000). 
Table 4

Representative vectors computed for the symbolic images shown in Fig. 11 in a sorted sequence

\begin{tabular}{lccl}
\hline Image index & First component & Second component & Third component \\
\hline P2 & -0.00560771 & 0.00050654 & 0.99998414 \\
P0 & -0.00439206 & 0.00031226 & 0.99999028 \\
P5 & -0.00375842 & 0.0004773 & 0.99999282 \\
P8 & -0.00361429 & 0.00425993 & 0.99998435 \\
P6 & -0.00314333 & 0.0028475 & 0.999991 \\
P9 & -0.00314333 & 0.0028475 & 0.999991 \\
P7 & -0.00304893 & 0.00065994 & 0.99999514 \\
P3 & -0.00183456 & -0.00192424 & 0.99999648 \\
P4 & -0.00092119 & -0.00102308 & 0.99999908 \\
P1 & 0.00556331 & 0.00558737 & 0.99996891 \\
\hline
\end{tabular}

Table 5

A sorted sequence of the representative vectors computed for all the symbolic images of Figs. 8, 10 and 11

\begin{tabular}{llcl}
\hline Image index & First component & Second component & Third component \\
\hline c & -0.00827159 & -0.00031386 & 0.99996576 \\
d & -0.00810757 & -0.00033761 & 0.99996707 \\
a & -0.00752611 & -0.00031277 & 0.99997163 \\
P2 & -0.00560771 & 0.00050654 & 0.99998414 \\
P0 & -0.00439206 & 0.00031226 & 0.99999028 \\
P5 & -0.00375842 & 0.0004773 & 0.99999282 \\
P8 & -0.00361429 & 0.00425993 & 0.99998435 \\
F3 & -0.00316463 & -0.00079141 & 0.99999467 \\
P6 & -0.00314333 & 0.0028475 & 0.999991 \\
P7 & -0.00304893 & 0.00065994 & 0.99999514 \\
F2 & -0.0021145 & 0.00185542 & 0.99999604 \\
P3 & -0.00183456 & -0.00192424 & 0.99999648 \\
b & -0.00143076 & -0.00094487 & 0.99999854 \\
P4 & -0.00092119 & -0.00102308 & 0.99999908 \\
F1 & 0.00182135 & 0.00457998 & 0.99998784 \\
F4 & 0.00214189 & 0.000336708 & 0.99999204 \\
P1 & 0.00556331 & 0.00558737 & 0.99996891 \\
\hline
\end{tabular}

\section{Discussion and conclusion}

Perception and representation of invariant spatial relationships existing among objects present in an image indeed helps in preserving the reality being embedded in an image. Devising schemes which are invariant, fast, flexible and good in preserving the reality being embedded in the images is a challenging task in the field of image databases. Infact, these are the shortcomings existing in almost all so far proposed methodologies.

Similarity match and exact match retrieval are the two major issues related to any image database. Similarity retrieval task deals with retrieving all those images that are similar to the given query image from the SID, while exact match retrieval process retrieves from SID, only those images, exactly identical to the query image and is more likely an image recognition problem.

Though, many models were devised for similarity retrieval, only a few attempts were made to devise invariant models for similarity retrieval. The similarity retrieval models claimed as invariant to image transformations are less efficient from the point of view of their usage for exact match retrieval as exact match retrieval can be achieved more efficiently and more effectively with less computation effort and less resource investment when compared to that of similarity retrieval. To 
the best of our knowledge only one model has been proposed for exact match retrieval but it is not invariant to image transformations specifically to rotation.

In view of this, in this paper, we have made a successful attempt in exploring a model which overcomes the aforementioned shortcomings and best suits exact match retrieval. The paper presents a way of representing a symbolic image in SID invariant to image transformations through the perception of spatial relationships. The newly introduced concept of direction of reference helps in perceiving pairwise spatial relationships among the components present in an image, invariant to image transformations. The first PCV obtained for the set of triples which preserves the perceived invariant spatial relationships, is stored in a sorted sequence as the representative of the image. The exact matched image is then retrieved through the application of modified binary search technique. The proposed model integrates the representation of an image with the retrieval of an image. Unlike, other models, our model automatically takes care of additional information such as angles as it is based on the direction of reference.

The transformation of a set of triples to the first PCV for different set of triples is not biunivocal, as there may be the same PCV generated for different sets of triples. Due to the limitation of PC transformation, there is a possibility of getting identical first PCVs for different symbolic images. Hence, two entirely different images may have the same PCV. Under such conflicting situations, one could employ second PCV for resolution, and go up to the third PCV. If all the PCVs associated with two or more symbolic images are same then it is suggested to compare the eigenvalues associated with the images. Upon its failure, it becomes inevitable to further compare the retrieved triples with that of the query image for exact identification. Furthermore, the precision of real numbers should be carefully handled on conducting a binary search in practical programming, since, each element of a PCV is a real number.

Unlike the 9DLT based approach (Chang and $\mathrm{Wu}, 1995)$, our method is invariant to image transformations and it takes care of multiple instances of objects, which is considered to be a major problem in most of the existing methodologies. In addition, our method requires $\mathrm{O}(\log n)$ search time in the worst case to retrieve an exact matched image from the SID.

A comparison of the proposed model with some of the other models is given in Table 6 .

It should be noticed that the proposed methodology concentrates only on exact match retrieval of symbolic images from a SID. The task of transforming a physical image into its corresponding symbolic image is itself a research topic (Chang and $\mathrm{Wu}, 1995)$. However, a few interesting attempts towards the transformation of physical

Table 6

Comparison of the proposed method with other previously proposed methodologies

\begin{tabular}{llll}
\hline $\begin{array}{l}\text { Representation and } \\
\text { retrieval schemes }\end{array}$ & Adopted data structure & $\begin{array}{l}\text { Invariant to image } \\
\text { transformation }\end{array}$ & Retrieval time complexity \\
\hline $\begin{array}{l}\text { Chang et al. (1987) } \\
\text { Lee et al. (1989) }\end{array}$ & 2D string & Not invariant & Non-polynomial \\
Lee and Hsu (1990) & 2D string & Not invariant & Non-polynomial \\
Lee and Hsu (1992) & 2D string & Not invariant & Non-polynomial \\
Chang and Wu (1992) & 2D-C string & Not invariant & $\mathrm{O}\left(m^{2}\right)$ \\
Wu and Chang (1994) & 2D string + hashing & Not invariant & $\mathrm{O}\left(m^{2}\right)$ \\
Chang and Wu (1995) & 2D string + hashing & Not invariant & $\mathrm{O}(\log n)$ \\
Petraglia et al. (1996) & 9DLT matrix + PCA & Invariant & $\mathrm{Exponential}$ \\
Sabharwal and Bhatia (1997) & 2D-R string & Not invariant & $\mathrm{O}\left(m^{2}\right)$ \\
Zhou and Ang (1997) & 2D string + hashing & Not invariant & $\mathrm{O}\left(m^{2}\right)$ \\
Zhou et al. (2001) & 9DLT matrix + hashing & Invariant & $\mathrm{O}\left(n^{2}\right)$ \\
The Proposed Scheme & A square matrix & Invariant & $\mathrm{O}(\log n)$ \\
\hline
\end{tabular}


image to a corresponding symbolic image can be found in (Guru, 2000).

The proposed model perceives the spatial relationships existing among the components present in the image represented in the form of symbols, regardless of the features and feature size of the components present in the image. Devising a methodology, which makes use of such features of the components for more efficient representation and retrieval, is a topic of our future work. Since, exact match retrieval is more likely an image recognition problem, our method appears to be useful for object recognition. Hence, our other goal is to adapt the proposed method for object recognition purpose.

In summary, an invariant model for exact match retrieval of symbolic image from a SID is proposed in this paper. The major problem in the 9DLT matrix based approaches is discussed. Unlike Chang and Wu's (1995) method, the proposed model is invariant to image transformations and the beauty of our scheme lies in its efficiency from the point of view of retrieval time (as it is of logarithmic time complexity). The efficacy of the proposed methodology is experimentally established by considering a large database of 10,800 images.

\section{Appendix A}

\section{Algorithm: Find first PCV \\ Input: Samples \\ Output: First PCV \\ Method:}

1. Find the variance-covariance matrix say $C$ of the samples.

2. Find the eigenvectors of $C$.

3. Choose the eigenvector corresponding to the largest eigenvalue as the first PCV.

\section{Algorithm ends}

\section{References}

Bhatia, S.K., Sabharwal, C.L., 1994. A fast implementation of a perfect hash function for picture objects. Pattern Recognition 27 (3), 365-375.
Chang, C.C., 1991. Spatial match retrieval of symbolic pictures. J. Informat. Sci. Eng. 7, 405-422.

Chang, Y.I., Ann, H.Y., 1999. A note on adaptive 2D-H strings. Pattern Recognition Lett. 20, 15-20.

Chang, C.C., Jiang, J.H., 1994. A fast spatial match retrieval using a superimposed coding technique. In: Proc. Internat. Symposium on Advanced Database Technologies and their Integration, Nara, Japan, pp. 71-78.

Chang, S.K., Li, Y., 1988. Representation of multi-resolution symbolic and binary pictures using 2D H strings. In: Proc. IEEE Workshop on Languages for Automata, pp. 190195.

Chang, C.C., Lin, D.C., 1996. A spatial data representation: An adaptive 2D H string. Pattern Recognition Lett. 17, 175185.

Chang, C.C., Wu, T.C., 1992. Retrieving the most similar symbolic pictures from pictorial databases. Informat. Process. Manage. 28 (5), 581-588.

Chang, C.C., Wu, T.C., 1995. An exact match retrieval scheme based upon principal component analysis. Pattern Recognition Lett. 16, 465-470.

Chang, S.K., Shi, Q.Y., Yan, C.W., 1987. Iconic indexing by 2D strings. IEEE Trans. Pattern Anal. Machine Intell. 9 (5), 413-418.

Chang, S.K., Jungert, E., Li, Y., 1989. Representation and retrieval of symbolic pictures using generalized 2D string. In: SPIE Proc. on Visual Comm. and Image Process., Philadelphia, pp. 1360-1372.

Guru, D.S., 2000. Towards accurate recognition of objects employing a partial knowledge base: Some new approaches. Ph.D. Thesis, Department of Studies in Computer Science, University of Mysore, Manasagangothri, Mysore, India.

Guru, D.S., Raghavendra, H.J., Suraj, M.G., 2000. An adaptive binary search based sorting by insertion: An efficient and simple algorithm. J. Statist. Appl. 2, 85-96.

Huang, P.W., Jean, Y.R., 1994. Using 2D $\mathrm{C}^{+}$strings as spatial Knowledge representation for image database systems. Pattern Recognition 27 (9), 1249-1257.

Jungert, E., 1988. Extended symbolic projection used in a knowledge structure for spatial reasoning. In: Proc. 4th BPRA Conf. on Pattern Recognition. Springer-Verlag.

Lee, S.Y., Hsu, F.J., 1990. 2D C string: A new spatial knowledge representation for image database system. Pattern Recognition 23 (10), 1077-1087.

Lee, S.Y., Hsu, F.J., 1991. Picture algebra for spatial reasoning of iconic images represented in $2 \mathrm{D} \mathrm{C}$ string. Pattern Recognition Lett. 12 (7), 425-435.

Lee, S.Y., Hsu, F.J., 1992. Spatial reasoning and similarity retrieval of images using 2D C string knowledge representation. Pattern Recognition 25 (3), 305-318.

Lee, S.Y., Shan, M.K., 1990. Access methods of image databases. Internat. J. Pattern Recognition Artificial Intell. 4 (1), 27-44.

Lee, S.Y., Shan, M.K., Yang, W.P., 1989. Similarity retrieval of iconic image databases. Pattern Recognition 22 (6), 675682. 
Petraglia, G., Sebillo, M., Tucci, M., Tortora, G., 1996. A normalized index for image databases. In: Chang, S.K., Jungert, E., Tortora, G. (Eds.), Intelligent Image Database Systems. World Scientific, Singapore.

Sabharwal, C.L., Bhatia, S.K., 1995. Perfect hash table algorithm for image databases using negative associated values. Pattern Recognition 28 (7), 1091-1101.

Sabharwal, C.L., Bhatia, S.K., 1997. Image databases and near perfect hash table. Pattern Recognition 30 (11), 1867-1876.
Wu, T.C., Chang, C.C., 1994. Application of geometric hashing to iconic database retrieval. Pattern Recognition Lett. 15, 871-876.

Zhou, X.M., Ang, C.H., 1997. Retrieving similar pictures from pictorial database by an improved hashing table. Pattern Recognition Lett. 18, 751-758.

Zhou, X.M., Ang, C.H., Ling, T.W., 2001. Image retrieval based on object's orientation spatial relationship. Pattern Recognition Lett. 22, 469-477. 